\title{
Cancer et Afrique
}

\section{Cancer and Africa}

\section{R. Salamon}

C Springer-Verlag France 2010

On peut aborder le cancer sous quatre aspects :

- diminuer son incidence par des actions de prévention primaires à l'encontre de facteurs de risque avérés et au fort impact ;

- le prendre en charge précocement lorsque cela est possible grâce à un dépistage approprié ;

- le diagnostiquer et le traiter avec les outils les mieux adaptés ;

- enfin, permettre au patient atteint de cancer de vivre une vie de la meilleure qualité possible.

Ces quatre temps imposent des démarches multisectorielles parfois complexes :

- la prévention primaire nécessite :

- une bonne connaissance des facteurs de risque ;

- des actions allant de l'éducation pour la santé individuelle ou collective jusqu'à des réglementations lourdes en matière industrielle, environnementale ou publicitaire ;

- le dépistage fait appel à des outils de détection fiables, performants, peu coûteux et acceptables pour une population cible bien définie.

Cela impose aussi :

- une action de promotion et de suivi du dépistage pour atteindre un taux suffisant de participation;

- et surtout, trop souvent oubliée, la mise en place de structures diagnostiques et thérapeutiques capables de prendre en charge les cas dépistés.

Rien n'est plus absurde et triste que de mettre en place des outils de dépistage performants sans s'assurer d'une prise en charge des cas ainsi dépistés ;

- les outils diagnostiques et thérapeutiques nécessitent pour être efficaces des investissements lourds, des services adaptés et des capacités d'innovation ;

R. Salamon $(\bowtie)$

ISPED, université Victor-Segalen-Bordeaux-II,

146, rue Léo-Saignat, F-33076 Bordeaux cedex, France

e-mail : Roger.Salamo@isped.u-bordeaux2.fr
- la qualité de vie des patients atteints de cancer, pour être optimale, impose un minimum de moyens et de personnels.

L'énumération de cette liste d'outils nécessaires pour une lutte efficace contre le cancer témoigne, dès sa lecture, des difficultés auxquelles est confronté le continent africain où les ressources sont limitées, les structures de soins adaptées insuffisantes, les stratégies de dépistage bien compromises, la surveillance épidémiologique, la recherche et l'analyse des facteurs de risque encore insuffisamment développées.

Que faire alors pour améliorer la situation?

Il faut certainement imaginer une collaboration Nord-Sud pour que l'Afrique puisse, en outre, bénéficier des expériences du Nord (les succès comme les échecs) et d'une aide matérielle et en formation.

Il faut aussi que les tutelles « bienfaitrices » (de l'OMS jusqu'aux nombreuses ONG ou fondations) mettent le maximum d'efforts pour combattre le cancer en Afrique.

Pour cela, il faudra que de nombreux changements s'opèrent :

- d'une part, que l'on cesse de croire ou de faire croire que l'Afrique n'est soumise qu'aux fléaux des maladies infectieuses et parasitaires.

Il faut se convaincre de l'importance des maladies chroniques sur le continent africain et cesser de déséquilibrer les aides financières aux seuls profits du sida ou du paludisme, même s'il est indispensable de maintenir et même de renforcer l'appui à la lutte contre ces deux fléaux ;

- d'autre part, il faut ne plus accepter que ce qui est interdit en Europe soit permis dans les pays du Sud : je pense, bien sûr, à la lutte contre le tabac (interdiction des publicités ou de la cigarette dans les lieux publics) mais aussi à de nombreuses autres mesures dans les secteurs professionnels ou environnementaux qui ont été prises au Nord et tardent à l'être au Sud. Que les gouvernements africains comprennent l'importance du problème et le rôle et les devoirs qui sont les leurs. 
En France, deux présidents de la République successifs ont mis en place des Plans cancers multisectoriels et ambitieux. Cela pourrait être un excellent modèle.

Enfin, la formation, la recherche et la surveillance épidémiologique doivent être améliorées, et les Africains doivent y devenir les acteurs principaux. Dans ce domaine, la revue « Journal africain du cancer » joue un rôle important dont il faut se féliciter.

Mais la tâche est immense, et les progrès seront lents. Raison de plus pour ne pas tarder. 